\title{
BOUNDS OF THE GINI INDEX USING SPARSE INFORMATION ON MEAN INCOMES
}

\author{
BY TOMSON OGWANG* \\ Economics Program, University of Northern British Columbia
}

In this paper, bounds of the Gini index, based on grouped data, are proposed assuming sparse information on mean incomes in the sense that data on either the overall mean income or some of the group mean incomes are not reported. It turns out that the proposed bounds are identical to those proposed by other authors that have more stringent information requirements with respect to mean incomes.

\section{INTRODUCTION}

Confidentiality, political sensitivity and other considerations often dictate the reporting of income inequality data by governments and other agencies in grouped format. In these cases, inequality measures, such as the Gini index, must be estimated from the grouped data rather than from the individual observations from which the grouped data are constructed. Also, grouped data, which are generally more widely available than micro-data, are particularly useful for cross-country studies of income inequality.

Several approaches to the measurement of the Gini index based on grouped data have been adopted in the literature. One parametric approach entails fitting a theoretical Lorenz curve (LC) to the grouped data and the Gini index (and in some cases the underlying density) is then deduced from the estimated parameters of the LC. See, for example, Kakwani and Podder (1973, 1976), Villaseñor and Arnold (1989), Ogwang and Rao (1996), Sarabia (1997), and Sarabia et al. (1999). Schader and Schmid (1994) have provided a fairly comprehensive survey of more than ten parametric LCs. Another parametric approach applies interpolation methods to deduce estimates of the Gini index, assuming the observed points on the LC to be fixed. See, for example, Gastwirth (1975), Gastwirth and Glauberman (1976), Cowell and Mehta (1982), and Brown and Mazzarino (1984).

More than two decades ago, Gastwirth (1972), Mehran (1975), Murray (1978), and Fuller (1979) proposed non-parametric approaches by deriving lower and upper bounds, from grouped data, within which the Gini index must lie regardless of the functional form of the underlying distribution of income. The appeal of these non-parametric approaches stems from the fact that no assumption regarding the shape of the underlying LC (or the corresponding income distribution) is necessary. The lower bound assumes that the incomes in each bracket are equally distributed whereas the upper bound also incorporates a "grouping

Note: I wish to thank Fiona MacPhail, Baotai Wang, Christopher Opio, Danny Cho, and anonymous referees for their valuable comments. However, the usual disclaimer applies.

*Correspondence to: Tomson Ogwang, Economics Program, University of Northern British Columbia, 3333 University Way, Prince George, BC, Canada V2N 4Z9 (ogwang@unbc.ca). 
correction factor" that generally assumes maximum inequality in that bracket. Gastwirth et al. (1986) have derived the joint asymptotic distribution of Gastwirth's lower and upper bounds, which can be used as a basis for forming confidence intervals for the population Gini index.

In practical applications, the size of the estimated bounds depends on the number of groups into which the incomes are divided as well as the degree of "richness" of information pertaining to the income brackets (Gastwirth, 1972, p. 311; Cowell, 1991). Gastwirth's upper bound requires knowledge of the limits of the income brackets, all the group mean incomes, as well as the overall mean income. Mehran's upper bound, on the other hand, does not require knowledge of the group mean incomes, the overall mean income or the limits of the income brackets. Fuller's upper bound, like Murray's upper bound, requires knowledge of the limits of the income brackets and the overall mean income but it cannot be computed if data on either the overall mean income or some of the group mean incomes are not reported. ${ }^{1}$ Clearly, Mehran's upper bound has the least stringent information requirements with respect to mean incomes and could be used in the absence of information on group mean incomes as well as the overall population mean income. However, if the data on group mean incomes or the overall mean income are provided with the grouped data, it seems sensible to exploit this information in the computation of the bounds of the Gini index.

The main purpose of this paper is to derive bounds of the Gini index from grouped data assuming the availability of sparse information on mean incomes in the sense that data on either the overall mean income or some of the group mean incomes are not reported. The proposed method exploits the relationships among the group mean incomes, the overall mean income, the slopes of line segments joining the observed points on the LC, and the slopes of tangents to the LC at the observed points. The resulting bounds turn out to be identical to both Gastwirth's and Fuller's bounds, which have more stringent information requirements with respect to mean incomes, but are smaller than Mehran's bounds which have less stringent information requirements. However, the relationship between the proposed bounds and Murray's bounds, that involve solution to linear programming problems (in the case of the lower bound) and quadratic programming problems (in the case of the upper bound), is not apparent.

The format of the rest of the paper is as follows. In Section 2, a brief description of some properties of the LC is provided. In Section 3, the proposed bounds are presented and their estimation using sparse information on mean incomes is discussed. In Section 4, the equivalence of the proposed bounds to Gastwirth's and Fuller's bounds is established. An illustrative example is provided in Section 5 , and Section 6 contains some concluding remarks.

\section{Properties of THE LC}

The LC is a convenient graphical device for studying the size distribution of income and wealth. It is defined by a set of ordered points $(p, L(p))$, where $p$ is

\footnotetext{
${ }^{1}$ Murray's upper bound also assumes that the upper income bracket is unbounded from above and the number of income receiving units in each income bracket is known.
} 
the cumulative proportion of the income-receiving units (e.g. persons, households) and $L(p)$, the corresponding cumulative proportion of income received, when the units are arranged in ascending order of income.

It is well known (e.g. Gastwirth, 1971, p. 1037; Kendall and Stuart, 1977, p. 48) that if a certain variable $a$ representing the level of income (where $a \geq 0$ ) is represented by a continuous probability distribution with density function, $f(a)$, cumulative distribution function, $F(a)$, and has a finite positive mean $\mu$, the associated LC is defined by the following two equations:

$$
\begin{gathered}
p=F(a)=\int_{0}^{a} f(a) d a \\
L(p)=\Phi(a)=\frac{1}{\mu} \int_{0}^{a} a f(a) d a
\end{gathered}
$$

where $\mu=\int_{0}^{\infty} a f(a) d a$ is the mean of $a$.

Equation (1) gives the proportion of income receiving units with incomes less than or equal to $a$. Equation (2) gives the proportion of income received by the income receiving units with incomes less than or equal to $a$. The LC is defined by plotting the values of $p$ and $L(p)$ in equations (1) and (2), respectively, corresponding to several arbitrary values of $a$.

The following two properties of the LC are noteworthy:

Property 1: The slope of the LC, as defined by equations (1) and (2) above, is given by

$$
\frac{d L(p)}{d p}=\frac{d F(a)}{d \Phi(a)}=\frac{a}{\mu}
$$

which increases as $a$ increases (i.e. the LC is monotonically non-decreasing in $p$ ). Furthermore, $\frac{d^{2} L(p)}{d p^{2}}>0$, establishing convexity of the LC toward the $p$-axis. From equation (3), it can be seen that the slope of the LC at any given level of income, say, $a_{i}$, which is, in fact, the slope of the tangent to the LC corresponding to income level $a_{i}$, is equal to $a_{i} / \mu$, where $\mu$ is the mean income.

Property 2: The Gini index, $G$, is given by twice the area between the LC and the perfect equality (egalitarian) line or, equivalently, as one minus twice the area under the LC, i.e.

$$
G=2 \int_{0}^{1}[p-L(p)] d p=1-2 \int_{0}^{1} L(p) d p
$$

\section{Descriptions of the Proposed Bounds}

The proposed bounds take the observed points on the LC computed from the grouped data as given. To provide explicit expressions for the proposed bounds, let us assume that the data are divided into $k+1$ income brackets with $a_{0}, a_{1}, a_{2}$, $\ldots, a_{k+1}$ as the interval endpoints $0 \leq a_{1}<a_{2}<\ldots<a_{k+1} \leq \infty$. For the upper bound to be valid, we also have to assume that the sample is drawn from a continuous distribution. Let $\mu_{i}, \mu, p_{i}$, and $L\left(p_{i}\right)$ denote the mean income in the $i$-th income 


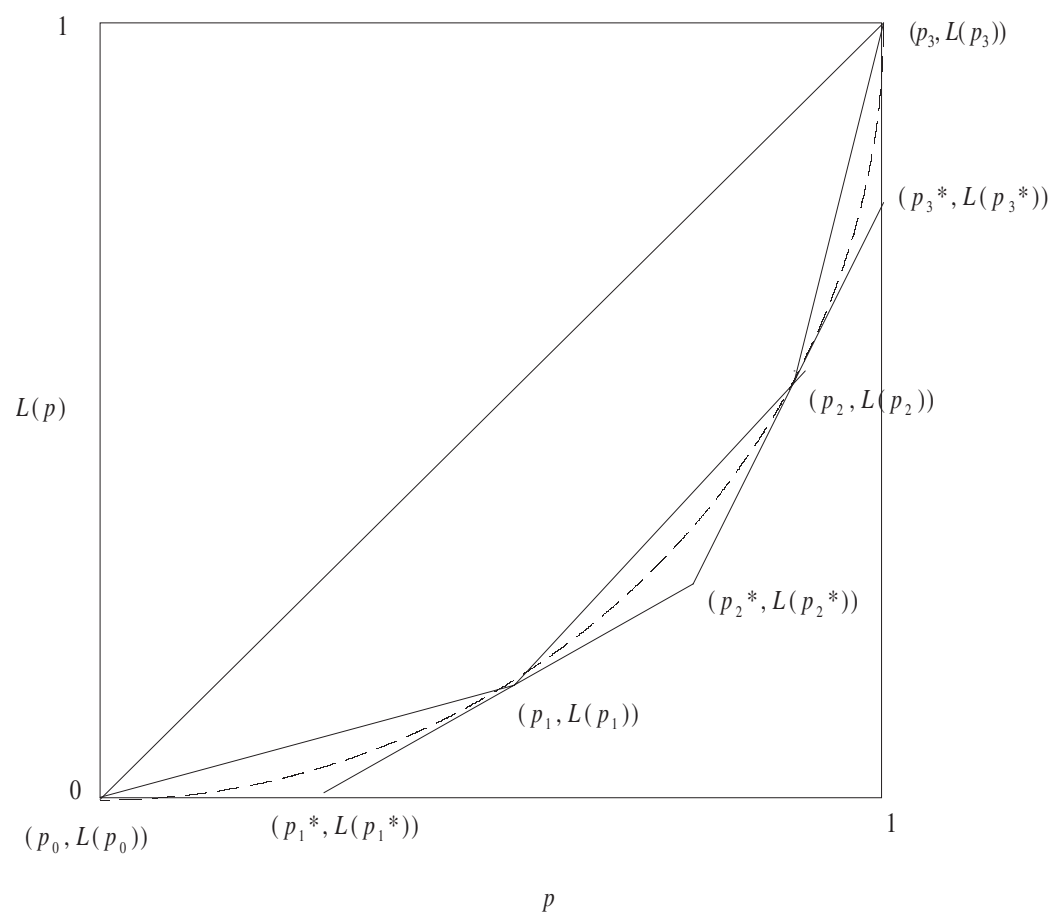

Figure 1. Lorenz curve coordinates assuming three income brackets

bracket $\left(a_{i-1}, a_{i}\right)$, the overall mean income, the cumulative fraction of income receiving units with income less than $a_{i}$, and the corresponding cumulative fraction of income, respectively. The corresponding LC is defined by a set of ordered points $\left(p_{i}, L\left(p_{i}\right)\right), i=0,1,2, \ldots, k+1$ as illustrated by the dashed convex curve in Figure 1 assuming three income brackets (i.e. $k+1=3)$. By definition, $\left(p_{0}, L\left(p_{0}\right)\right)$ $=(0,0)$ and $\left(p_{k+1}, L\left(p_{k+1}\right)\right)=(1,1)$. The perfect equality (egalitarian) line is the line segment joining $(0,0)$ and $(1,1)$.

Let $\beta_{i}$ be the slope of the line segment joining $\left(p_{i-1}, L\left(p_{i-1}\right)\right)$ and $\left(p_{i}, L\left(p_{i}\right)\right)$ i.e. $\beta_{i}=\frac{\left(L\left(p_{i}\right)-L\left(p_{i-1}\right)\right)}{\left(p_{i}-p_{i-1}\right)} i=1,2, \ldots, k+1$. Also, let $\beta_{i}^{*}$ be the slope of the tangent to the LC at $\left(p_{i}, L\left(p_{i}\right)\right)$ and let $\left(p_{i}^{*}, L\left(p_{i}^{*}\right)\right)$ be the point of intersection of the tangents to the LC at $\left(p_{i}, L\left(p_{i}\right)\right)$ and $\left(p_{i-1}, L\left(p_{i-1}\right)\right)$ as demonstrated in Figure 1. It is not difficult to verify that ${ }^{2} \beta_{0}^{*}=0, \beta_{i}^{*}=\frac{\left(L\left(p_{i}\right)-L\left(p_{i}^{*}\right)\right)}{\left(p_{i}-p_{i}^{*}\right)} i=1,2, \ldots, k$ and $\beta_{k+1}^{*}=\infty$.

Furthermore, $p_{i}^{*}=\frac{\left(\beta_{i}^{*} p_{i}-\beta_{i-1}^{*} p_{i-1}\right)-\beta_{i}\left(p_{i}-p_{i-1}\right)}{\left(\beta_{i}^{*}-\beta_{i-1}{ }^{*}\right)} i=1,2, \ldots, k$, and $p_{k+1} *=1$. Also, $L\left(p_{1}^{*}\right)=0, L\left(p_{i}^{*}\right)=L\left(p_{i-1}^{*}\right)+\beta_{i-1}^{*}\left(p_{i}^{*}-p_{i-1}^{*}\right) i=2, \ldots, k$, and $L\left(p_{k+1}^{*}\right)=$ $\beta_{k}^{*}\left(p_{k+1}-p_{k}\right)+L\left(p_{k}\right)$.

${ }^{2}$ Detailed derivations are available from the author on request. 
Obtaining the lower bound of the Gini index entails determining the area enclosed by the sequence of line segments joining the observed points on the LC (i.e. $\left.\left(p_{i}, L\left(p_{i}\right)\right) i=0,1,2, \ldots, k+1\right)$ and the perfect equality line and multiplying the result by two (see Property 2 above). The resulting estimate of the Gini index defines the lower bound since this sequence of line segments lies inside or on the LC, thus exhibiting a lower degree of income inequality than that depicted by the LC itself. The upper bound of the Gini index is obtained by determining the area formed by the tangents to the LC at all the observed points on the LC (i.e. $\left(p_{i}^{*}\right.$, $\left.\left.L\left(p_{i}^{*}\right)\right) i=1,2, \ldots, k+1\right)$ and the perfect equality line and multiplying the result by two. The resulting estimate of the Gini index defines the upper bound since these tangents include areas outside the LC, thus exhibiting a higher degree of income inequality than that depicted by the LC itself.

The proposed lower bound of the Gini index, $L$, which is identical to Gastwirth's lower bound, is given by

$$
L=1-\sum_{i=1}^{k+1} \beta_{i}^{-1}\left(L\left(p_{i}\right)^{2}-L\left(p_{i-1}\right)^{2}\right)
$$

If the top income bracket is bounded from above, the proposed upper bound is given by

$$
U_{O 1}=L+\sum_{i=1}^{k+1}\left(p_{i}-p_{i-1}\right)^{2}\left(\beta_{i}^{*}-\beta_{i}\right)\left(\beta_{i}-\beta_{i-1}^{*}\right)\left(\beta_{i}^{*}-\beta_{i-1}^{*}\right)^{-1}
$$

Practical applications of the proposed method require estimates of $\beta_{i}^{*} i=1$, $2, \ldots, k+1 .^{3}$ It follows from equation (3) that the upper bound of the Gini index can be obtained by substituting $\beta_{i}^{*}=a_{i} / \mu i=1,2, \ldots, k+1$, into equation (6a), where $\mu$ is the overall mean income.

If the top income bracket is not bounded from above (i.e. $a_{k+1}=\infty$ ), the proposed upper bound is given by

$$
\begin{aligned}
U_{O 2}= & L+\left[\sum_{i=1}^{k}\left(p_{i}-p_{i-1}\right)^{2}\left(\beta_{i}^{*}-\beta_{i}\right)\left(\beta_{i}-\beta_{i-1}^{*}\right)\left(\beta_{i}^{*}-\beta_{i-1}^{*}\right)^{-1}\right] \\
& +\left(\beta_{k+1}-\beta_{k}^{*}\right)\left(p_{k+1}-p_{k}\right)^{2}
\end{aligned}
$$

where $p_{k+1}=1$.

The terms augmenting the $L$ on the right hand side of equations (6a) and (6b) represent grouping correction factors. It is apparent from equations (3), (6a) and (6b) that computation of the proposed upper bound requires knowledge of the overall mean income. If the overall mean income is not reported but all the group mean incomes are reported, recovery of the overall mean income using information on the (cumulative) proportion of the income receiving units in each income bracket is a trivial matter. However, problems arise if the overall mean income and

${ }^{3}$ Although similar results were derived by Mehran (1975), the proposed bounds differ in the way the $\beta_{i}^{*}$ 's are constructed. Specifically, Mehran's bounds are based on the choice of the estimates of the $\beta_{i}^{*}$ 's that maximize the grouping correction factors, which may yield a slightly bigger upper bound than the proposed upper bound. 
some of the group mean incomes are not reported. We will now address the problem of computing the upper bound of the Gini index using equation (6a) or (6b), in the absence of information on some of the group mean incomes, by first recovering the overall mean income. Specifically, we will show how the proposed bounds can still easily be computed provided that accurate information on at least one of the group mean incomes is reported. To see this, let us consider the following additional property of the LC.

Property 3: If $\mu$ is the overall mean income and $\beta_{i} i=1,2, \ldots, k+1$ are the slopes of the line segments joining the observed points on the LC as defined above, then the mean income in the $i$-th income bracket (i.e. income bracket $\left(a_{i-1}, \alpha_{i}\right)$ as defined above) is given by

$$
\mu_{i}=\beta_{i} \mu
$$

Equation (7) can be used to recover the group mean incomes from the overall mean income, and vice versa, once the $\beta_{i}$ 's are computed from the observed points on the LC.

Clearly, there are two situations under which sparse information on mean incomes can be used to determine the upper bound of the Gini index using the proposed method. First, if the overall mean income is known, the bounds of the Gini index can be computed using equation (6a) or (6b) even though information on the group mean incomes is not provided. Second, if the overall mean is not known but at least one of the group means is known accurately, equation (7) can be used to recover the overall mean income from one of the reported group mean incomes which is, in turn, used to compute the bounds of the Gini index. Thus, we have demonstrated, in the context of the Gini index, how different degrees of "richness" of information pertaining to mean incomes can, in fact, yield identical bounds.

\section{Equivalence Between the Proposed Bounds And GASTWIRTH'S/FULLER'S BOUNDS}

If the top income bracket is bounded from above, Gastwirth's upper bound of the Gini index is given by

$$
U_{G 1}=L+\mu^{-1} \sum_{i=1}^{k+1}\left(p_{i}-p_{i-1}\right)^{2}\left(a_{i}-\mu_{i}\right)\left(\mu_{i}-a_{i-1}\right)\left(a_{i}-a_{i-1}\right)^{-1}
$$

If the top income bracket is not bounded from above, Gastwirth's upper bound is given by

$$
\begin{aligned}
U_{G 2}= & L+\left[\mu^{-1} \sum_{i=1}^{k+1}\left(p_{i}-p_{i-1}\right)^{2}\left(a_{i}-\mu_{i}\right)\left(\mu_{i}-a_{i}\right)\left(a_{i}-a_{i-1}\right)^{-1}\right] \\
& +\mu^{-1}\left(p_{k+1}-p_{k}\right)^{2}\left(\mu_{k+1}-a_{k}\right)
\end{aligned}
$$

where $p_{k+1}=1$ as indicated above.

To demonstrate the equivalence between the proposed upper bound (equation (6a)) and Gastwirth's upper bound (equation (8a)), we note that equation (6a) can be written as 


$$
\begin{aligned}
U_{O 1} & =L+\sum_{i=1}^{k+1}\left(p_{i}-p_{i-1}\right)^{2}\left(\frac{a_{i}}{\mu}-\beta_{i}\right)\left(\beta_{i}-\frac{a_{i-1}}{\mu}\right)\left(\frac{a_{i}}{\mu}-\frac{a_{i-1}}{\mu}\right)^{-1} \\
& =L+\sum_{i=1}^{k+1}\left(p_{i}-p_{i-1}\right)^{2}\left(\frac{a_{i}-\beta_{i} \mu}{\mu}\right)\left(\frac{\beta_{i} \mu-a_{i-1}}{\mu}\right)\left(\frac{a_{i}-a_{i-1}}{\mu}\right)^{-1} \\
& =L+\mu^{-1} \sum_{i=1}^{k+1}\left(p_{i}-p_{i-1}\right)^{2}\left(a_{i}-\mu_{i}\right)\left(\mu_{i}-a_{i-1}\right)\left(a_{i}-a_{i-1}\right)^{-1} \\
& =U_{G 1} \quad \text { (Equation 8a) }
\end{aligned}
$$

Note that in deriving the above result, we have made use of the fact that $\beta_{i}^{*}=a_{i} / \mu$ and $\mu_{i}=\beta_{i} \mu$. A similar procedure can be followed to verify the equivalence of equation (6b) to equation ( $8 b)$.

If the top income bracket is bounded from above, Fuller's upper bound for the Gini index is given by

(9a) $U_{F 1}=L+\sum_{i=1}^{k+1} \frac{\left[\left(L\left(p_{i}\right)-L\left(p_{i-1}\right)\right)-\frac{a_{i-1}}{\mu}\left(p_{i}-p_{i-1}\right)\right]\left[\frac{a_{i}}{\mu}\left(p_{i}-p_{i-1}\right)-\left(L\left(p_{i}\right)-L\left(p_{i-1}\right)\right)\right]}{\left(\frac{a_{i}}{\mu}-\frac{a_{i-1}}{\mu}\right)}$

If the top income bracket is not bounded from above, Fuller's upper bound is given by

$$
\begin{aligned}
U_{F 2}= & L+\left\{\sum_{i=1}^{k+1} \frac{\left[\left(L\left(p_{i}\right)-L\left(p_{i-1}\right)\right)-\frac{a_{i-1}}{\mu}\left(p_{i}-p_{i-1}\right)\right]\left[\frac{a_{i}}{\mu}\left(p_{i}-p_{i-1}\right)-\left(L\left(p_{i}\right)-L\left(p_{i-1}\right)\right)\right]}{\left(\frac{a_{i}}{\mu}-\frac{a_{i-1}}{\mu}\right)}\right\} \\
& +\left[\left(L\left(p_{k+1}\right)-L\left(p_{k}\right)\right)-\frac{a_{k}}{\mu}\left(p_{k+1}-p_{k}\right)\right]\left[p_{k+1}-p_{k}\right]
\end{aligned}
$$

The equivalence of the proposed upper bound to Fuller's upper bound can easily be established by using the fact that in equations (9a) and $(9 \mathrm{~b})\left(L\left(p_{i}\right)-\right.$ $\left.L\left(p_{i-1}\right)\right)=\beta_{i}\left(p_{i}-p_{i-1}\right)$ and $\beta_{i}^{*}=a_{i} / \mu$. In summary, the proposed upper bound, Gastwirth's upper bound, and Fuller's upper bound do use the same grouping correction factors, yet they have different information requirements with respect to mean incomes. Clearly, the result of these comparisons is that some of the information requirements are redundant.

\section{AN Illustrative EXAMPLE}

To illustrate the computation of the proposed bounds, we used the same data originally collected by the United States Bureau of the Census, which were previously used by Gastwirth (1972) and Mehran (1975) to demonstrate the computations of their respective bounds. One important characteristic of this data set is that both the overall mean income and all the group mean incomes are known. For details pertaining to these data that are divided into ten income brackets with 
TABLE 1

Computations Pertaining to The Proposed Bounds*

\begin{tabular}{rcccccccrr}
\hline \hline$i$ & $a_{i}\left(x 10^{3}\right)$ & $p_{i}$ & $L\left(p_{i}\right)$ & $\beta_{i}$ & $\beta_{i}{ }^{*}$ & $L\left(p_{i}{ }^{*}\right)$ & $p_{i}{ }^{*}$ & $\mu_{i}($ given $)$ & $\mu_{i}($ estimated $)$ \\
\hline 0 & 0 & 0.00000 & 0.00000 & - & 0.00000 & - & - & - & - \\
1 & 1 & 0.04824 & 0.00300 & 0.06700 & 0.12351 & 0.0000 & 0.0221 & 541.41 & 541.1097 \\
2 & 2 & 0.13077 & 0.01820 & 0.18078 & 0.24702 & 0.0090 & 0.0925 & $1,463.63$ & $1,463.6894$ \\
3 & 3 & 0.20292 & 0.03990 & 0.30201 & 0.37054 & 0.0280 & 0.1708 & $2,445.72$ & $2,445.1914$ \\
4 & 4 & 0.27194 & 0.06930 & 0.42466 & 0.49405 & 0.0543 & 0.2417 & $3,438.90$ & $3,438.2133$ \\
5 & 5 & 0.33809 & 0.10550 & 0.54800 & 0.61756 & 0.0877 & 0.3092 & $4,437.32$ & $4,436.8027$ \\
6 & 6 & 0.41407 & 0.15618 & 0.66702 & 0.74107 & 0.1336 & 0.3836 & $5,401.18$ & $5,400.4416$ \\
7 & 7 & 0.49254 & 0.21813 & 0.78947 & 0.86458 & 0.1915 & 0.4618 & $6,392.92$ & $6,391.8947$ \\
8 & 10 & 0.70658 & 0.43763 & 1.02551 & 1.23512 & 0.3228 & 0.6136 & $8,304.54$ & $8,302.9331$ \\
9 & 15 & 0.89769 & 0.71857 & 1.47004 & 1.85268 & 0.5839 & 0.8250 & $11,904.33$ & $11,902.0596$ \\
10 & $\infty$ & 1.00000 & 1.00000 & 2.75076 & $\infty$ & 0.9081 & 1.0000 & $22,261.50$ & $22,271.2330$ \\
\hline
\end{tabular}

*Notes: $\mu=8,096.4$.

In column $6 \beta_{i}^{*}=a_{i} / \mu$; in column $10, \mu_{i}$ (estimated $)=\beta_{i} \mu$.

The definitions of all the variables are given in Section 3.

the top income bracket not bounded from above, see Gastwirth (1972, Table 2) and Mehran (1975, p. 66). The results of the computations involved are summarized in Table 1. The resulting estimates of the proposed lower and upper bounds are 0.3883 and 0.4083 , respectively, which are identical to the corresponding estimates of Gastwirth's bounds, based on the same data. ${ }^{4}$ In contrast, the estimate of Mehran's upper bound based on the same data is 0.4087 (Mehran, 1975, p. 66), which is bigger than the estimate of the proposed upper bound. The actual value of the Gini index, computed from the full sample of approximately 60,000 observations used to construct the grouped data, reported by Gastwirth (1972, p. 310), is 0.4014 which lies within the proposed bounds. ${ }^{5}$ The second last column of Table 1 shows the reported group mean incomes and the last column of the table shows the estimated group mean incomes, based on equation (7), assuming that the overall mean income is known but the group mean incomes are not. Except for rounding errors, the estimated group means are identical to the reported group means.

\section{CONCLUding REMARKS}

In this paper, bounds of the Gini index, based on grouped data, were proposed assuming sparse information on mean incomes. It turns out that the proposed bounds are identical to Gastwirth's and Fuller's bounds that have more stringent information requirements with respect to mean incomes. Finally, although the proposed method does not address sampling variability issues arising from the fact that the observed points on the LC are considered to be fixed (see, e.g. McDonald and Ransom, 1981), the method should prove useful in most income distribution studies that typically involve working with very large samples.

${ }^{4}$ Note that equation (6b) is used to compute the upper bound since the upper income bracket is not bounded from above. The lower bound is computed using equation (5).

${ }^{5}$ It would also have been interesting to compare the proposed bounds with Murray's bounds. However, the absence of information on the number of income receiving units in each group, and our inability to recover it from the observed points on the LC, precluded us from doing so. 


\section{REFERENCES}

Brown, J. A. C. and G. Mazzarino, "Drawing the Lorenz Curve and Calculating the Gini Concentration Index from Grouped Data by Computer," Oxford Bulletin of Economics and Statistics, 46, 273-8, 1984.

Cowell, Frank A. and Fatemeh Mehta, "The Estimation and Interpolation of Inequality Measures," Review of Economic Studies, 49, 273-90, 1982.

Cowell, Frank A., "Grouping Bounds for Inequality Measures Under Alternative Informational Assumptions," Journal of Econometrics, 48, 1-14, 1991.

Fuller, Mike, "The Estimation of Gini Coefficients from Grouped Data: Upper and Lower Bounds," Economics Letters, 3, 187-92, 1979.

Gastwirth, Joseph L., "A General Definition of the Lorenz Curve," Econometrica, 39, 1037-9, 1971. , "The Estimation of the Lorenz Curve and Gini Index," Review of Economics and Statistics, 54, 306-16, 1972.

_ "The Estimation of a Family of Measures of Economic Inequality," Econometrica, 42, 191-6, 1975.

Gastwisth, Joseph, L. and Marcia Glauberman, "The Interpolation of the Lorenz Curve and Gini Index from Grouped Data," Econometrica, 44, 479-83, 1976.

Gastwisth, Joseph, L., Tapan K. Nayak, and Abba M. Krieger, "Large Sample Theory for the Bounds on the Gini and Related Indices of Inequality Estimated From Grouped Data," Journal of Business and Economic Statistics, 4, 269-73, 1986.

Kakwani, N. C. and N. Podder, "On the Estimation of Lorenz Curves from Grouped Observations," International Economic Review, 14, 278-92, June 1973. , "Efficient Estimation of the Lorenz Curve and Associated Inequality Measures from Grouped Observations," Econometrica, 44, 137-48, January 1976.

Kendall, Maurice G. and Alan Stuart, The Advanced Theory of Statistics, 1, Griffin, London, 1977.

McDonald, James B. and Michael R. Ransom, "An Analysis of the Bounds for the Gini Coefficient," Journal of Econometrics, 17, 177-88, 1981.

Mehran, Farhad, "Bounds on the Gini Index Based on Observed Points of the Lorenz Curve," Journal of the American Statistical Association, 70, 64-6, March 1975.

Murray, David, "Extreme Values for Gini Coefficients Calculated from Grouped Data," Economics Letters, 1, 389-93, 1978.

Ogwang, Tomson and U. L. Gouranga Rao, "A New Functional Form for Approximating the Lorenz Curve," Economics Letters, 52, 21-9, 1996.

Sarabia, José-María, "A Hierarchy of Lorenz Curves Based on the Generalized Tukey's Lambda Distribution," Econometric Reviews, 16, 305-20, 1997.

, Enrique Castillo and Daniel J. Slottje, "An Ordered Family of Lorenz Curves," Journal of Econometrics, 91, 43-60, 1999.

Schader, Martin and Friedrich Schmid, "Fitting Parametric Lorenz Curves to Grouped Income Distributions-A Critical Note,” Empirical Economics, 19, 361-70, 1994.

Villaseñor, José A. and Barry C. Arnold, "Elliptical Lorenz Curves," Journal of Econometrics, 40, 327-38, 1989. 